\title{
Validation of the Tuebingen CD-25 Inventory as a Measure of Postoperative Health-Related Quality of Life in Patients Treated for Cushing's Disease
}

\author{
Monika Milian $^{a}$ Ilonka Kreitschmann-Andermahr $^{b}$ Sonja Siegel $^{d}$

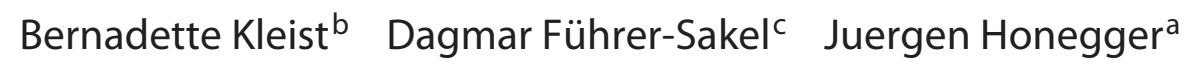 \\ Michael Buchfelder ${ }^{d}$ Tsambika Psaras ${ }^{e}$ \\ ${ }^{a}$ Department of Neurosurgery, University Hospital Tuebingen, Tuebingen, Departments of ${ }^{b}$ Neurosurgery and \\ 'Endocrinology, University Hospital Essen, Essen, ${ }^{\mathrm{d}}$ Department of Neurosurgery, University Hospital Erlangen, \\ Erlangen, and ${ }^{e}$ Department of Neurosurgery, Cologne-Merheim Hospital, Cologne, Germany
}

\section{Key Words}

Cushing's disease · Validity · Tuebingen Cushing's

disease quality of life inventory · Cushing's quality of life

questionnaire $\cdot$ Short Form 36

\section{Abstract \\ Background: To evaluate the construct and criterion validity of the Tuebingen Cushing's disease quality of life inventory (Tuebingen CD-25) for application in patients treated for Cushing's disease (CD). Methods: A total of 176 patients with adrenocorticotropin hormone-dependent CD (144 of them female, overall mean age $46.1 \pm 13.7$ years) treated at 3 large tertiary referral centers in Germany were studied. Construct validity was assessed by hypothesis testing (self-perceived symptom reduction assessment) and contrasted groups (pa- tients with vs. without hypercorticolism). For this purpose, already existing data from 55 CD patients was used, repre- senting the hypercortisolemic group. Criterion validity (con- current validity) was assessed in relation to the Cushing's quality of life questionnaire (CushingQoL), the Short Form 36 health survey (SF-36), and the body mass index (BMI). $\boldsymbol{R e}$ - sults: Patients with self-perceived remarkable symptom re-}

duction had significant lower Tuebingen CD-25 scores (i.e. better health-related quality of life) than patients with selfperceived insufficient symptom reduction $(p<0.05)$. Similarly, the mean scores of the Tuebingen CD- 25 scales were lower in patients without hypercortisolism (total score 27.0 \pm 17.2 ) compared to those with hypercortisolism (total score $45.3 \pm 22.1$; each $p<0.05$ ), providing evidence for construct validity. Criterion validity was confirmed by the correlations between the Tuebingen CD-25 total score and the CushingQoL (Spearman's coefficient -0.733), as well as all scales of the SF-36 (Spearman's coefficient between -0.447 and -0.700). Conclusion: The analyses presented in this large-sample study provide robust evidence for the construct and criterion validity of the Tuebingen CD- 25 .

(c) 2015 S. Karger AG, Basel

\section{Introduction}

Cushing's disease (CD) is a rare pathology resulting from chronic exposure to hypercortisolism due to an adrenocorticotropin hormone-secreting pituitary corticotroph adenoma [1]. Patients with CD suffer not only from

\section{KARGER 125}

(c) 2015 S. Karger AG, Basel

0028-3835/15/1022-0060\$39.50/0

E-Mail karger@karger.com

www.karger.com/nen
Monika Milian

Department of Neurosurgery, University of Tuebingen

Hoppe Seyler Strasse 3

DE-72076 Tuebingen (Germany)

E-Mail monika.milian@med.uni-tuebingen.de 
visible external changes but also from metabolic, cardiovascular, cognitive, and psychiatric comorbidities. CD is, therefore, a condition that obviously has severe effects on patients' health-related quality of life (HRQoL) [2]. Impairments concerning the HRQoL have been increasingly recognized as an important aspect of clinical management in patients suffering from CD [3], as HRQoL among $\mathrm{CD}$ patients is reduced compared to the general population. Approximately $80 \%$ of patients with active CD suffer from impairments in HRQoL compared to age- and sex-matched healthy controls $[4,5]$. Postoperatively, one third of all CD patients continue to suffer from impaired HRQoL despite biochemical remission [5], which is attributed to irreversible effects caused by glucocorticoid excess [6].

Before the development of the Cushing's quality of life questionnaire (CushingQoL) [7] in 2008, HRQoL and psychosocial impairment were assessed using a variety of non-disease-specific questionnaires, e.g. the Short Form 36 (SF-36) health survey [8] or the Hospital Anxiety and Depression Scale (HADS) [9]. However, all of these questionnaires have the disadvantage that they may miss certain disease-specific aspects of importance. In 2012, we developed the second CD-specific HRQoL inventory, i.e. the Tuebingen Cushing's disease quality of life inventory (Tuebingen CD-25), in order to have a German-language inventory and a multidimensional instrument. The instrument covers 6 domains of HRQoL in $\mathrm{CD}$ (i.e. mood, sexual activity, social environment, eating behavior, physical aspects, and cognitive functioning) and provides a total score [10]. The inventory has shown excellent internal consistency (Cronbach's $\alpha=0.94$ ) and a high validity (correlation coefficients between -0.65 and -0.74$)[5,10]$, determined by the correlations between the total score of the Tuebingen $\mathrm{CD}$ 25 and the overall score of the established WHO Quality of Life-BREF (WHOQoL-BREF [11]). However, to date, no validity data about the Tuebingen CD-25 against the widely used CushingQoL and the most widely used generic tool for assessing HRQoL, i.e. the SF-36, is available.

The primary aim of the present study was to obtain validity data for the Tuebingen CD-25 in a very large sample of treated CD patients. The objectives were to provide evidence of: (1) construct validity by means of hypothesis testing (self-perceived symptom reduction) and contrasted groups (disease status), and (2) criterion validity through concurrence (correlations with standard measurements).

Validity of the Tuebingen CD-25

Inventory
Table 1. Demographic and clinical characteristics of the $176 \mathrm{CD}$ patients

\begin{tabular}{ll}
\hline Variables & Measures \\
\hline Age, years & $46.1 \pm 13.7$ \\
Male/female ratio & $144(81.8 \%) / 32(18.2 \%)$ \\
Time between symptom onset and CD & \\
$\quad$ diagnosis, years & $3.8 \pm 4.8$ \\
Time between CD diagnosis and study & \\
$\quad$ participation, years & $7.6 \pm 7.5$ \\
BMI & $27.3 \pm 6.3$ \\
\hline
\end{tabular}

Values are presented as means \pm SD unless otherwise stated.

\section{Materials and Methods}

\section{Patients}

We report on 176 patients with CD (144 females and 32 males) who were surgically treated at 3 large tertiary referral centers in Germany between 1983 and 2013. One hundred one CD patients were treated at the University Hospital Erlangen, 44 were treated at the University Hospital Tuebingen, and 31 were treated at the University Hospital Essen. All patients were contacted via mail and asked to complete a self-developed questionnaire that covered their sociodemographic background, the history of the disease, and the medical treatment modalities of their disease, as well as the Tuebingen CD-25 and the SF-36. For the 44 patients treated in Tuebingen, CushingQoL data was also available due to an addition to the study protocol.

The clinical characteristics, diagnostic processes, and demographic data (education and work status) have been reported elsewhere [12] and are summarized here (table 1).

The mean age of the 176 patients at the time of their study participation was $46.1 \pm 13.7$ years (range $18-88$ ). The mean time between symptom onset and establishment of the diagnosis of CD was $3.8 \pm 4.8$ years. The mean time between $\mathrm{CD}$ diagnosis and study participation was $7.6 \pm 7.5$ years. Patients were operated on, on average, 1.4 times. When asked about their last measured level of cortisol, $44.3 \%$ of the patients $(n=78)$ stated that the level was normal, $17.0 \%(\mathrm{n}=30)$ stated that the level was low, and $5.7 \%$ $(\mathrm{n}=10)$ stated that level was still elevated. Fifty-eight patients (33.0\%) could not provide precise information about their cortisol level. Thus, 108 patients were considered nonhypercortisolemic for further analyses.

Hydrocortisone replacement therapy was necessary in $52.3 \%$ of all patients, growth hormone in $8.0 \%$, thyroid hormones in $39.2 \%$, and sex hormones in $14.2 \%$. Antihypertensive medication was taken by $29.0 \%$, antidepressants by $14.8 \%$, and diabetes medication by $11.9 \%$ of the patients. Postoperative radiotherapy was performed in $13.6 \%$ of the patients.

The research protocol was approved by the Ethics Committee of the University of Erlangen-Nuremberg (Erlangen, Germany), the Ethics Committee of the University of Tuebingen (Tuebingen, Germany), and the Ethics Committee of the University of EssenDuisburg (Essen, Germany) and was performed under the tenets 
of the Declaration of Helsinki and its subsequent amendments. Written informed consent was given by all patients prior to participating in this study.

\section{Instruments}

The following instruments were used in the research protocol:

\section{Tuebingen CD-25}

For the development of the Tuebingen CD-25 [10] technical literature, a board of experienced endocrinologists, pituitary surgeons, and a neuropsychologist, as well as previously treated patients with CD, were consulted. For the development of the present inventory, a preliminary inventory comprising 64 HRQoL items was handed out to $63 \mathrm{CD}$ patients. Subsequent item reduction was performed by means of the classical test theory, including analysis of items, internal consistency, and patterns of correlation, as well as confirmatory factorial analysis. Twenty-five items remained in the final version of the questionnaire. The Tuebingen CD-25 covers 6 essential HRQoL dimensions in CD: (1) depression, (2) sexual activity, (3) environment, (4) eating behavior, (5) bodily restrictions, and (6) cognition. The mean completion time is $10 \mathrm{~min}$, which is highly desirable as it allows use in both research and realtime clinical settings.

Response options are given on a 5-point Likert type scale from 0 (strongly disagree) to 4 (strongly agree). In order to be able to compare the different subscales, it has been predetermined that each subscale and the total score have a minimum score of 0 and a maximum score of 100 , with higher scores representing a lower QoL. The summed item raw scores for each subscale form a raw subscale score which then has to be transformed via a simple linear transformation by applying the following formula:

$$
T S=\frac{\text { attained } R S(\text { of the respective subscale })}{\text { max PS }(\text { of the respective subscale })} \times 100 \text {, }
$$

where TS is the transformed score, RS is the raw score, and PS is the possible score (each scale has another possible score).

The total score of the inventory is theoretically obtained using the same formula; however, a transformation for the total score is not necessary since the maximum raw score is - due to the 25 items - already 100. Thus, in the case of the total score the raw score corresponds to the transformed score.

\section{Cushing's Quality of Life Questionnaire}

The CushingQoL [7] is the first developed and validated disease-specific HRQoL inventory for CD consisting of 12 items on a 5 -point Likert scale. The total score ranges from 12 to 60 and is converted to a scale of $0-100$, with 0 indicating the worst HRQoL and 100 indicating the best HRQoL.

\section{Short-Form Health Survey}

The SF-36 [8] questionnaire comprises 36 items and records general well-being. The items are formulated as statements or questions to assess 8 health concepts: (1) limitations in physical activities because of health problems (physical functioning), (2) limitations in usual role activities because of physical health problems (physical role), (3) pain (bodily pain), (4) general health perceptions and changes in health (general health), (5) energy and fatigue (vitality), (6) limitations in social activities because of phys- ical or emotional problems (social functioning), (7) limitations in usual role activities because of emotional problems (emotional role), and (8) psychological distress and well-being (mental health). Transformed scores are expressed on a scale of $0-100$ points, with higher scores associated with a better quality of life.

\section{Body Mass Index}

The BMI was determined from the demographic and medical history questionnaire based on the patients' mass and body height. The BMI was shown to be a predictor of HRQoL in treated CD patients [13].

\section{Demographic and Medical History Questionnaire}

A survey was conducted to assess information on cohort characteristics. Patients received a self-developed 51-item questionnaire. Next to covering sociodemographic data (e.g. age, level of education, marital and occupational status, weight, and height), the course of diagnostic process, the time from the onset of symptoms to CD diagnosis, the time between CD diagnosis and study participation, treatment, and perception of symptom reduction after surgical treatment were assessed. Self-perceived symptom reduction estimation was included for construct validation and was evaluated based on 4 answer choices (no reduction, slight reduction, strong reduction, and complete reduction).

Additionally, patients were asked to indicate whether the last measured cortisol value was classified as low, normal, or elevated in order to identify the group of cured patients. This group was included for construct validation (contrasted groups) in relation to the group of hypercortisolemic patients (for further information, see Evaluation Methods).

\section{Evaluation Methods}

The 176 patients completed all of the questionnaires at the same time. Analyses were based on classical psychometric methods. The evidence for construct validity (hypothesis testing) was based on responses to the question: 'How strong have your symptoms decreased by the medical treatment of CD (no reduction, slight reduction, strong reduction and complete reduction)?' by investigating whether there were group differences with respect to the scores. Further verification of construct validity (contrast groups) was obtained by means of HRQoL comparisons in relation to the current disease status (patients without vs. with hypercortisolism). Since the group of hypercortisolemic patients $(\mathrm{n}=10)$ in the present sample was too small for statistical comparisons, we included the data of 43 nonredundant patients from the Tuebingen CD-25 development study $[4,10]$ and the data of 12 recently diagnosed hypercortisolemic (i.e. after admission for neurosurgical treatment) CD patients from the Department of Neurosurgery in Tuebingen, amounting to a total sample size of 55 hypercortisolemic CD patients. Of these, 44 were female and 11 were male; the mean age was $50.5 \pm 15.9$ years. Their data was compared to that of the 108 nonhypercortisolemic patients from the present cohort. Both groups were similar concerning gender distribution $\left(\chi^{2}[1]=0.465, \mathrm{p}=0.495\right)$.

Evidence of criterion validity (concurrent validity) was obtained by examining the correlation of the Tuebingen CD-25 with the SF-36 and the CushingQoL. Additionally, the concurrent validity of the eating behavior scale of the Tuebingen CD-25 was assessed by correlating the score with the BMI. The correlations between the Tuebingen CD-25 and the SF-36 and the CushingQoL, 
Table 2. Mean HRQoL scores in the $176 \mathrm{CD}$ patients

\begin{tabular}{ll}
\hline Variables & Scores \\
\hline Tuebingen CD-25 & \\
$\quad$ Depression & $27.1 \pm 22.7$ \\
Sexual activity & $34.8 \pm 27.1$ \\
Environment & $30.9 \pm 22.8$ \\
Eating behavior & $22.6 \pm 22.6$ \\
Bodily restrictions & $28.3 \pm 25.9$ \\
Cognition & $40.2 \pm 30.9$ \\
Total score & $29.2 \pm 18.5$ \\
CushingQoL & $58.1 \pm 17.9$ \\
SF-36 & \\
Physical functioning & $71.8 \pm 26.5$ \\
Physical role & $55.7 \pm 42.7$ \\
Bodily pain & $65.8 \pm 30.4$ \\
General health & $53.9 \pm 22.9$ \\
Vitality & $45.8 \pm 19.8$ \\
Social functioning & $70.5 \pm 27.9$ \\
Emotional role & $64.5 \pm 43.3$ \\
Mental health & $62.5 \pm 21.2$ \\
\hline
\end{tabular}

Values are presented as means $\pm \mathrm{SD} .{ }^{\text {a }}$ Data from 44 patients is available.

respectively, were hypothesized to be negative and those with the BMI were expected to be positive. Correlations were defined based on Spearman's coefficient as follows: highly strong, coefficient $>0.70$; strong, coefficient $0.5-0.7$; moderate, coefficient $0.30-0.50$, and weak, coefficient $<0.3$.

\section{Statistical Methods}

Data were analyzed using SPSS (Statistical Package for the Social Sciences) version 21.0 for Windows. Descriptive statistics were used to analyze sociodemographic and clinical characteristics (e.g. age, time to diagnosis, and time since diagnosis) using means and standard deviations (SD) for parametric data. The number of patients was used for categorical variables (e.g. gender, education, and work status). Differences in gender distribution between 2 groups were evaluated using the $\chi^{2}$ test. The Kolmogorov-Smirnov test resulted in nonnormally distributed data for the Tuebingen CD-25, the SF-36, and the BMI. A multivariate analysis of variance (MANOVA) was used to determine whether there were any differences between the 4 symptom reduction groups (i.e. no reduction, a slight reduction, a strong reduction, or complete reduction) on the scales of the Tuebingen CD-25 (hypothesis testing). Then, we followed up the significant ANOVAs with Tukey's honestly significant difference post hoc tests (multiple comparisons). Regarding comparisons between patients without hypercortisolism and those with hypercortisolism, to evaluate contrasted group validity, a MANOVA was used as well subsequent univariate ANOVAs. Due to the nonnormal distribution, Spearman's rank correlation coefficients were calculated to evaluate concurrent validity by correlating the Tuebingen CD-25 with standard measurements. $\mathrm{p}<$ 0.05 was considered statistically significant.

Validity of the Tuebingen CD-25 Inventory

\section{Results}

The detailed HRQoL data of the 176 treated patients is presented in table 2 . In the present cohort, the mean total score of the Tuebingen CD-25 was $29.2 \pm 18.5$. Regarding explicitly the patients who reported being eucortisolemic or hypocortisolemic $(n=108)$, the mean total score on the Tuebingen CD-25 was $27.0 \pm 17.2$, comparable to that in a previous study [5], with a mean score of $27.6 \pm 20.6$ in cured CD patients.

\section{Construct Validity: Hypothesis Testing}

The MANOVA revealed a significant multivariate main effect for self-perceived symptom reduction [Wilks' $\lambda=0.743, \mathrm{~F}(21,414.04)=2.148, \mathrm{p}<0.01]$. Given the significance of the overall test, the univariate main effects were examined. Significant univariate main effects for self-perceived symptom reduction were obtained for all subscales and the total score on the Tuebingen CD-25. The results of the univariate ANOVA tests are shown in table 3.

Post hoc analyses with the Tukey honestly significant difference test showed that mean depression scores were statistically significantly different between the groups no reduction and strong reduction $(\mathrm{p}<0.01)$ and no reduction and complete reduction ( $\mathrm{p}<0.01)$, but not between no reduction and slight reduction $(\mathrm{p}=0.299)$ or strong reduction and complete reduction $(\mathrm{p}=0.404)$. Mean sexual activity scores were statistically significantly different between the groups slight reduction and strong reduction $(\mathrm{p}<0.05)$ and slight reduction and complete reduction $(\mathrm{p}<0.01)$, but not between no reduction and slight reduction $(\mathrm{p}=1.000)$ or strong reduction and complete reduction ( $p=0.863)$. In the scale environment, patients with no self-perceived symptom reduction had significant higher scores (i.e. a lower HRQoL) compared to patients with self-perceived complete symptom reduction $(\mathrm{p}<$ $0.05)$. Moreover, mean environment scores were statistically significantly different between the groups slight reduction and strong reduction $(\mathrm{p}<0.05)$ and slight reduction and complete reduction ( $\mathrm{p}<0.001)$, but not between no reduction and slight reduction $(\mathrm{p}=1.000)$. However, there was a clear trend for a significant difference between the groups strong reduction and complete reduction $(\mathrm{p}=$ $0.051)$.

Regarding the eating behavior scale, patients with a self-perceived slight reduction had a significant lower HRQoL compared to patients with a self-perceived complete symptom reduction $(\mathrm{p}<0.01)$. Mean bodily restriction scores were statistically significantly different be- 
Table 3. Construct validity (hypothesis testing): mean Tuebingen CD-25 scores by self-perceived symptom reduction

\begin{tabular}{|c|c|c|c|c|c|}
\hline & \multicolumn{5}{|c|}{ Symptom reduction } \\
\hline & $\begin{array}{l}\text { none } \\
(\mathrm{n}=12)\end{array}$ & $\begin{array}{l}\text { slight } \\
(\mathrm{n}=33)\end{array}$ & $\begin{array}{l}\text { strong } \\
(\mathrm{n}=84)\end{array}$ & $\begin{array}{l}\text { complete } \\
(\mathrm{n}=34)\end{array}$ & $\mathrm{p}$ value \\
\hline \multicolumn{6}{|l|}{ Tuebingen CD-25 } \\
\hline Depression & $48.7 \pm 14.9$ & $37.5 \pm 28.2$ & $24.1 \pm 18.0$ & $17.2 \pm 20.7$ & 0.000 \\
\hline Sexual activity & $46.4 \pm 31.1$ & $49.2 \pm 30.0$ & $30.9 \pm 24.9$ & $26.8 \pm 20.8$ & 0.003 \\
\hline Environment & $42.3 \pm 20.6$ & $44.0 \pm 24.9$ & $28.6 \pm 20.2$ & $17.6 \pm 18.1$ & 0.000 \\
\hline Eating behavior & $20.8 \pm 17.7$ & $34.5 \pm 31.8$ & $22.1 \pm 20.3$ & $13.8 \pm 16.1$ & 0.011 \\
\hline Bodily restrictions & $40.3 \pm 24.0$ & $41.6 \pm 31.9$ & $25.2 \pm 24.2$ & $21.2 \pm 21.2$ & 0.014 \\
\hline Cognition & $55.1 \pm 31.4$ & $53.8 \pm 31.3$ & $37.9 \pm 29.7$ & $25.4 \pm 26.9$ & 0.001 \\
\hline Total score & $40.6 \pm 14.5$ & $40.4 \pm 23.7$ & $27.2 \pm 15.2$ & $19.5 \pm 13.8$ & 0.000 \\
\hline
\end{tabular}

Of the 176 patients, 13 (7.4\%) provided no information on self-perceived symptom reduction. Values are presented as means \pm SD unless otherwise stated.

tween the groups slight reduction and strong reduction $(\mathrm{p}<0.05)$ and slight reduction and complete reduction $(\mathrm{p}<0.05)$, but not between no reduction and slight reduction ( $\mathrm{p}=0.961$ ) or strong reduction and complete reduction ( $\mathrm{p}=0.886)$. Regarding the cognition scale, patients with a self-perceived complete symptom reduction scored significantly better than patients with a self-perceived slight symptom reduction $(\mathrm{p}<0.01)$. There was also a clear trend towards significant differences between the groups no reduction and complete reduction $(\mathrm{p}=0.053)$ and slight reduction and strong reduction $(\mathrm{p}=0.062)$, but not between no reduction and slight reduction $(\mathrm{p}=1.000)$ or strong reduction and complete reduction $(\mathrm{p}=0.174)$.

For the total score of the Tuebingen CD-25, analyses showed that mean scores were statistically significantly different between the groups no reduction and complete reduction $(\mathrm{p}<0.01)$, slight reduction and strong reduction $(\mathrm{p}<0.01)$, and slight reduction and complete reduction $(\mathrm{p}<0.001)$, but not between no reduction and slight reduction $(\mathrm{p}=1.000)$ or strong reduction and complete reduction $(\mathrm{p}=0.131)$.

\section{Construct Validity: Contrasted Groups}

The results of the assessment of contrasted group validity performed on the Tuebingen CD-25 are shown in table 4 . The MANOVA revealed a significant multivariate main effect for current disease status [Wilks' $\lambda=0.742$, $F(7,154)=7.656, p<0.001]$. Significant univariate main effects for current disease status were obtained for all subscales and the total score on the Tuebingen CD-25 (each $\mathrm{p}<0.05)$, meaning that the mean scores on the Tuebing-
Table 4. Construct validity (contrasted groups) of the Tuebingen CD-25, with disease activity being the external anchor

\begin{tabular}{llll}
\hline Variables & $\begin{array}{l}\text { Patients without } \\
\text { hypercortisolism } \\
(\mathrm{n}=108)\end{array}$ & $\begin{array}{l}\text { Patients with } \\
\text { hypercortisolism } \\
(\mathrm{n}=55)^{\mathrm{a}}\end{array}$ & $\mathrm{p}$ \\
\hline $\begin{array}{l}\text { Tuebingen CD-25 } \\
\quad \text { Depression }\end{array}$ & $24.0 \pm 21.1$ & $41.7 \pm 27.4$ & 0.000 \\
$\quad$ Sexual activity & $33.0 \pm 26.3$ & $42.6 \pm 28.7$ & 0.036 \\
Environment & $28.0 \pm 21.9$ & $46.7 \pm 27.4$ & 0.000 \\
Eating behavior & $21.2 \pm 21.3$ & $41.8 \pm 24.9$ & 0.000 \\
Bodily restrictions & $24.7 \pm 23.4$ & $50.6 \pm 28.3$ & 0.000 \\
Cognition & $35.2 \pm 29.4$ & $55.9 \pm 26.4$ & 0.000 \\
Total score & $27.0 \pm 17.2$ & $45.3 \pm 22.1$ & 0.000 \\
\hline
\end{tabular}

${ }^{a}$ Data $(n=43)$ from the development study of the Tuebingen CD-25 and 12 recently recruited hypercortisolemic patients.

en CD-25 scales were significantly lower (i.e. a better HRQoL) in patients without hypercortisolism (total score $27.0 \pm 17.2$ ) compared to those with hypercortisolism (total score $45.3 \pm 22.1$ ).

\section{Criterion Validity: Concurrent}

As shown in table 5, the Tuebingen total score correlated with all scales of the SF-36 and the CushingQoL. Highly strong correlations (i.e. Spearman's coefficient $>0.70$ ) were found between the Tuebingen CD-25 total score and the CushingQoL score (Spearman's coefficient -0.733 ; fig. 1). Strong correlations (i.e. Spearman's coefficient $0.50-0.70$ ) were found between the Tuebingen 
Table 5. Criterion validity (concurrent validity) of the Tuebingen CD-25

\begin{tabular}{clllllll}
\hline \multicolumn{7}{c}{ Tuebingen CD-25 } \\
\cline { 2 - 8 } & Depression & $\begin{array}{l}\text { Sexual } \\
\text { activity }\end{array}$ & Environment & $\begin{array}{l}\text { Eating } \\
\text { behavior }\end{array}$ & $\begin{array}{l}\text { Bodily } \\
\text { restrictions }\end{array}$ & Cognition & $\begin{array}{l}\text { Total } \\
\text { score }\end{array}$ \\
\hline SF-36 & & & & & & & \\
PF & $-0.398^{* *}$ & $-0.221^{* *}$ & $-0.430^{* *}$ & $-0.197^{* *}$ & $-0.529^{* *}$ & $-0.418^{* *}$ & $-0.453^{* *}$ \\
RP & $-0.397^{* *}$ & -0.152 & $-0.458^{* *}$ & -0.148 & $-0.426^{* *}$ & $-0.484^{* *}$ & $-0.447^{* *}$ \\
BP & $-0.433^{* *}$ & $-0.248^{* *}$ & $-0.439^{* *}$ & $-0.201^{* *}$ & $-0.424^{* *}$ & $-0.398^{* *}$ & $-0.468^{* *}$ \\
GH & $-0.604^{* *}$ & $-0.355^{* *}$ & $-0.617^{* *}$ & $-0.172^{*}$ & $-0.461^{* *}$ & $-0.523^{* *}$ & $-0.620^{* *}$ \\
VT & $-0.655^{* *}$ & $-0.420^{* *}$ & $-0.635^{* *}$ & $-0.275^{* *}$ & $-0.462^{* *}$ & $-0.596^{* *}$ & $-0.700^{* *}$ \\
SF & $-0.622^{* *}$ & $-0.371^{* *}$ & $-0.560^{* *}$ & $-0.266^{* *}$ & $-0.409^{* *}$ & $-0.533^{* *}$ & $-0.621^{* *}$ \\
RE & $-0.533^{* *}$ & $-0.269^{* *}$ & $-0.412^{* *}$ & $-0.201^{* *}$ & $-0.366^{* *}$ & $-0.503^{* *}$ & $-0.489^{* *}$ \\
MH & $-0.698^{* *}$ & $-0.456^{* *}$ & $-0.597^{* *}$ & $-0.235^{* *}$ & $-0.446^{* *}$ & $-0.544^{* *}$ & $-0.678^{* *}$ \\
CushingQoL & $-0.773^{* *}$ & -0.189 & $-0.652^{* *}$ & $-0.322^{*}$ & $-0.566^{* *}$ & $-0.585^{* *}$ & $-0.733^{* *}$ \\
\hline
\end{tabular}

$\mathrm{PF}=$ Physical functioning; $\mathrm{RP}=$ physical role; $\mathrm{BP}=$ bodily pain; $\mathrm{GH}=$ general health; $\mathrm{VT}=$ vitality; $\mathrm{SF}=$ social functioning; $\mathrm{RE}=$ emotional role; $\mathrm{MH}=$ mental health. ${ }^{*} \mathrm{p}<0.05$ (significant); ${ }^{* *} \mathrm{p}<0.01$ (highly significant).

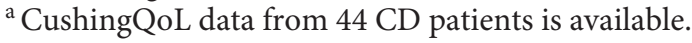

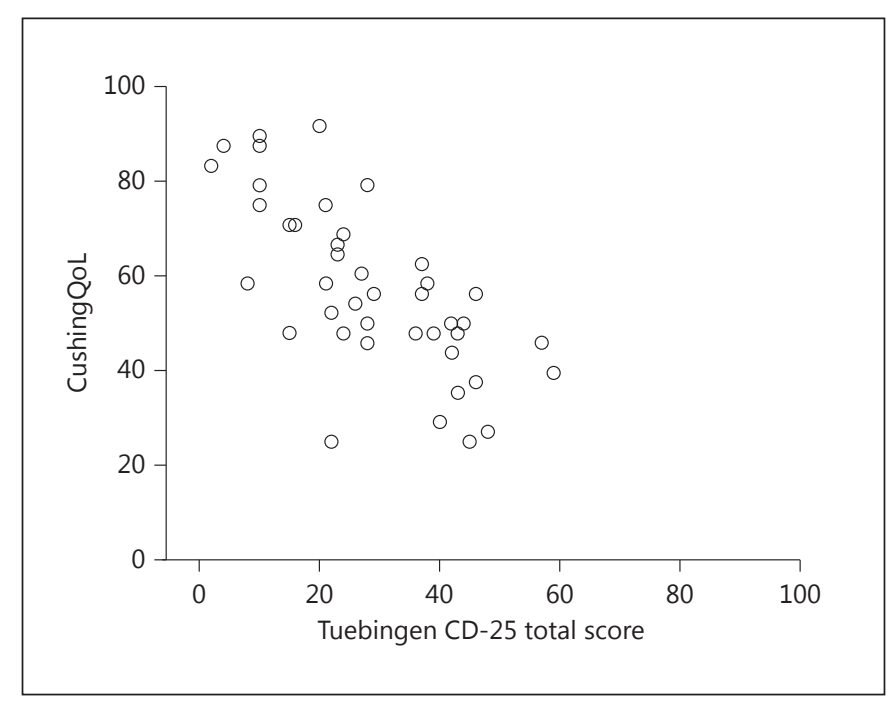

Fig. 1. Correlation between the Tuebingen CD-25 total score and the CushingQoL in 44 patients treated for CD (Spearman's coefficient -0.733).

CD-25 total score and the general health, vitality, social functioning, and mental health scales of the SF-36. Moderate correlations (i.e. Spearman's coefficient $0.30-0.50$ ) existed between the Tuebingen CD-25 total score and the physical functioning, physical role, bodily pain, and emotional role scales of the SF-36 survey. The subscales of the questionnaires, which are similar in content (e.g. the mental health scale of the SF-36 and the depression scale of the Tuebingen CD-25, Spearman's coefficient -0.698), correlated more strongly with each other than subdomains that were less related (e.g. the physical role scale of the SF-36 and the depression scale of the Tuebingen CD25 , Spearman's coefficient -0.397 ).

Scores on the eating behavior scale of the Tuebingen CD-25 correlated only weakly with the SF-36 scales (Spearman's coefficient -0.148 to -0.275 ). However, they correlated moderately with patients' BMI (Spearman's coefficient $0.441, \mathrm{p}<0.001)$. Interestingly, scores on the sexual activity scale were not significantly related to the CushingQoL score (Spearman's coefficient -0.189 ) but were related to the mental health scale of the SF-36 (Spearman's coefficient -0.456 ).

\section{Discussion}

For CD, 2 disease-specific HRQoL inventories are available: the CushingQoL [7] and the Tuebingen CD-25 [10]. Both questionnaires have shown in earlier studies a high reliability, validity, and sensitivity to change [5, 14]. The advantage of the CushingQoL is its short processing time ( $94 \%$ of patients complete the questionnaire in a mean time of $4 \mathrm{~min}$ ). The authors have also managed to find a mapping function which successfully predicts the EQ-5D [15] UK utilities from disease-specific CushingQOL scores [16]. To date, the quite recently developed Tuebingen CD-25 inventory has not been validated in a very large sample of treated CD patients. For this pur- 
pose, we collected data from $176 \mathrm{CD}$ patients who were surgically treated during the last 3 decades in 3 large tertiary referral centers in Germany.

The construct validity of the Tuebingen CD-25 was assessed via hypothesis testing as well as contrasted groups. For hypothesis testing, patients were asked to subjectively rate their symptom reduction. In fact, patients who reported an insufficient symptom reduction showed significant higher scores on the inventory (i.e. a lower HRQoL) compared to those who rated their symptom reduction as strong or complete. Construct validity was also confirmed by the contrasted group approach as patients with hypercortisolism showed a significantly lower HRQoL compared to those without hypercortisolism in all scales of the Tuebingen CD-25. Similar, in the study of Webb et al. [7], patients with current hypercortisolism scored worse than those without.

Evidence for criterion validity (concurrent) was provided by correlations between the Tuebingen CD-25 and other well-validated inventories. The total score correlated with all dimensions of the SF-36 (Spearman's coefficients between -0.447 and -0.700 ). Correlations were even higher when correlating the Tuebingen CD-25 with the previous standard, i.e. the CushingQoL, obtaining a Spearman's coefficient of -0.733 . As the SF- 36 is a generic questionnaire and was not designed to specifically target HRQoL in CD, it should be expected that the correlation to a disease-specific questionnaire, such as the CushingQoL, is higher.

Since the SF-36 and the CushingQoL do not cover the domain of patients' sexual activity and eating behavior, we only found none to weak correlations between these two scales of the Tuebingen CD-25 and the CushingQoL and the SF-36. However, in other diseases, e.g. multiple sclerosis [17] and diabetes [18], sexual dysfunction has been found to represent a substantial disease aspect that contributes negatively to the quality of life. Therefore, we assume that impaired sexual activity is a burden for CD patients as well. However, this aspect of CD is frequently not addressed, since clinicians commonly concentrate on the classical endocrinological deficits.

Concerning the eating behavior scale of the Tuebingen CD-25, we found that it was moderately correlated with the patients' BMI. A high BMI was shown to be a predictor for a worse HRQoL in CD [13]; therefore, we assume that questions covering eating behavior and body weight are relevant aspects in CD.

The Tuebingen CD inventory is, despite its high level of detail, suitable in a busy clinical surrounding since the questionnaire is self-explanatory and its completion re- quires no monitoring. Thus, patients can complete the inventory during their waiting time. Furthermore, determination of the total score requires simple mental arithmetic. Overall, 25 responses between 0 and 4 must be added and no further transformation for the total score (maximum $25 \times 4=100$ ) is mandatory since here the raw score corresponds to the transformed score. Concerning the subscales of the Tuebingen CD-25, a simple linear transformation is needed to obtain the transformed scores. Afterwards, by means of the transformed scores clinicians can easily determine whether a patient is affected or not by using the cut-off values (see the updated age- and sex-normed reference values from Milian et al. [4] published as corrigendum in 2013).

The advantages of the Tuebingen CD-25 compared to the CushingQoL are 3-fold: (1) the simple adding of the answers allows quick analysis of the total score since in this case no transformation is mandatory, (2) the inventory offers, beyond the total score, a more detailed view of the HRQoL due to the 6 subscales, including sexual activity and eating behavior, and (3) the normative data for the inventory allows interpretation of the scores. Thus, the inventory is suitable for application in clinical research, i.e. for evaluation of the therapeutic response and group comparisons in clinical trials, and in clinical practice for evaluating individual trends. Based on impairment of HRQoL for the different subdimensions, specific support can be offered to the patients. The disadvantages of the Tuebingen CD-25 compared to the CushingQoL are its longer completion (mean $10 \mathrm{~min}$ ) and evaluation time (in case that all subscales are evaluated separately). In addition, in contrast to the CushingQoL, the Tuebingen CD-25 does not include questions about bodily pain.

Whether the Tuebingen CD-25 questionnaire is also suitable for patients with Cushing's syndrome needs to be addressed in a further study. Although both pathologies manifest with the same clinical signs and symptoms, patients with CD may have different quality of life concerns, especially after long-term remission, because of having a tumor on the pituitary and going through brain surgery.

A limitation of the present study is that we could not report on hormone assessment in the group of $176 \mathrm{pa}$ tients since they were contacted by mail. Thus, the patients' information on cortisol levels was used for the assessment of disease activity. However, we assume that the information of the patients is valid since the other patients, who were not aware of their disease status, gave no information. Because the group of patients who reported suffering from elevated cortisol levels was too small for
Milian et al. 
statistical comparisons, we had to include further data from $55 \mathrm{CD}$ patients to investigate construct validity (contrasted group approach).

\section{Conclusion}

The analyses presented in this large sample of treated CD patients provide evidence for the construct and criterion validity of the Tuebingen CD-25. The results emphasize that disease-specific questionnaires should be used for valid HRQoL assessment in CD since generic questionnaires are prone to overlooking important aspects.

\section{Acknowledgement}

This work was supported by an independent investigatorinitiated grant from Novartis Oncology Germany (project No. RSIG-23).

\section{Disclosure Statement}

Tsambika Psaras, Ilonka Kreitschmann-Andermahr, Sonja Siegel, Dagmar Führer-Sakel, Jürgen Honegger, Michael Buchfelder, and Monika Milian have received research and/or travel grants and/or speaker honoraria from Novartis, Germany. The other authors report no conflict of interests.

\section{References}

1 Orth DN: Cushing's syndrome. N Engl J Med 1995;332:791-803.

-2 Feelders RA, Pulgar SJ, Kempel A, Pereira AM: The burden of Cushing's disease: clinical and health-related quality of life aspects. Eur J Endocrinol 2012;167:311-326.

-3 Sonino N, Bonnini S, Fallo F, Boscaro M, Fava GA: Personality characteristics and quality of life in patients treated for Cushing's syndrome. Clin Endocrinol 2006;64:314-318.

-4 Milian M, Teufel P, Honegger J, Gallwitz B, Schnauder G, Psaras T: The development of the Tuebingen Cushing's disease quality of life inventory (Tuebingen CD-25). 2. Normative data from 1,784 healthy people. Clin Endocrinol (Oxf) 2012;76:861-867.

5 Milian M, Honegger J, Teufel P, Wolf A, Psaras T: Tuebingen CD-25 is a sensitive tool to investigate health-related quality of life in Cushing's disease patients in the course of the disease. Neuroendocrinology 2013;98:188199.

-6 van Aken MO, Pereira AM, Biermasz NR, van Thiel SW, Hoftijzer HC, Smit JW, Roelfsema F, Lamberts SW, Romijn JA: Quality of life in patients after long-term biochemical cure of Cushing's disease. J Clin Endocrinol Metab 2005;90:3279-3286.

7 Webb SM, Badia X, Barahona MJ, Colao A, Strasburger CJ, Tabarin A, van Aken MO,
Pivonello R, Stalla G, Lamberts SW, Glusman JE: Evaluation of health-related quality of life in patients with Cushing's syndrome with a new questionnaire. Eur J Endocrinol 2008; 158:623-630.

8 Ware JE Jr, Sherbourne CD: The MOS 36item short-form health survey (SF-36). 1. Conceptual framework and item selection. Med Care 1992;30:473-483.

-9 Zigmond AS, Snaith RP: The hospital anxiety and depression scale. Acta Psychiatr Scand 1983;67:361-370.

10 Milian M, Teufel P, Honegger J, Gallwitz B, Schnauder G, Psaras T: The development of the Tuebingen Cushing's disease quality of life inventory (Tuebingen CD-25). 1. Construction and psychometric properties. Clin Endocrinol (Oxf) 2012;76:851-860.

11 Angermeyer MC, Kilian R, Matschinger $\mathrm{H}$ : WHOQoL-100 und WHOQoL-BREF Handbuch für die deutschsprachige Version der WHO Instrumente zur Erfassung von Lebensqualität. Göttingen, Hogrefe, 2000.

12 Kreitschmann-Andermahr I, Psaras T, Tsiogka M, Starz D, Kleist B, Siegel S, Milian M, Kohlmann J, Menzel C, Fuhrer-Sakel D, Honegger J, Sure U, Muller O, Buchfelder M: From first symptoms to final diagnosis of Cushing's disease: experiences of 176 patients. Eur J Endocrinol 2015;172:285-289.
13 Carluccio A, Sundaram NK, Chablani S, Amrock LG, Lambert JK, Post KD, Geer EB: Predictors of quality of life in 102 patients with treated Cushing's disease. Clin Endocrinol (Oxf) 2015;82:404-411.

14 Santos A, Resmini E, Martinez-Momblan MA, Crespo I, Valassi E, Roset M, Badia X, Webb SM: Psychometric performance of the CushingQoL questionnaire in conditions of real clinical practice. Eur J Endocrinol 2012; 167:337-342.

15 Rabin R, de Charro F: EQ-5D: a measure of health status from the EuroQol Group. Ann Med 2001;33:337-343.

16 Badia X, Roset M, Valassi E, Franz H, Forsythe A, Webb SM: Mapping CushingQoL scores to EQ-5D utility values using data from the European Registry on Cushing's Syndrome (ERCUSYN). Qual Life Res 2013;22: 2941-2950.

17 Lew-Starowicz M, Rola R: Sexual dysfunctions and sexual quality of life in men with multiple sclerosis. J Sex Med 2014;11:12941301.

18 Pozzo MJ, Mociulsky J, Martinez ET, Senatore G, Farias JM, Sapetti A, Sanzana MG, Gonzalez P, Cafferata A, Peloche A, Lemme L: Diabetes and quality of life: initial approach to depression, physical activity, and sexual dysfunction. Am J Ther 2014, E-pub ahead of print. 